\title{
Indemnité de déplacement pour le Congrès des Sociétés savantes
}

Acsp-Réunion annuelle générale, Memorial University, St. John's, Terre-Neuve, 8-10 juin 1997

L'Association canadienne de science politique reçoit des fonds du Conseil de recherches en sciences humaines du Canada pour les frais de déplacement des membres résidant dans les régions qui sont loin de la conférence. Ces subventions s'adressent avant tout aux membresparticipants les moins établis. On a tout lieu de croire que les professeurs recevront l'aide de leur université. Les bénéficiaires seront tous désignés avant le ler mars. L'Acsp suggère aux requérants et requérantes de prendre note des critères suivants car ils sont appliqués à chaque demande de bourse.

1) Les requérants et requérantes doivent être membres de l'Association canadienne de science politique et/ou de la Société québécoise de science politique pendant deux ans consécutives avant la demande de bourse.

2) Les bourses ont trait à des déplacements au sein de l'Amérique du Nord; elles seront calculées au prorata en fonction des meilleurs tarifs aériens offerts chaque année. Pour permettre d'ajuster les bourses de l'Acsp en fonction des besoins, les requérants et requérantes sont tenus de dévoiler à l'Association toute autre bourse/subvention dont ils bénéficient pour le même voyage. Toute portion de la bourse qui dépasse les tarifs aériens doit être remise à l'Acsp le plus rapidement possible afin de permettre une nouvelle répartition des fonds.

3) L'ordre de priorité régissant l'octroi des bourses sera le suivant : tout conférencier ou toute conférencière faisant partie des deux catégories suivantes (maximum: une bourse par exposé); a) les membres qui n'ont pas obtenu leur permanence; et b) les étudiants et étudiantes sauf ceux avec emploi à temps plein. La priorité sera accordée aux étudiants et étudiantes au doctorat avec projet de recherche avancé.

4) La priorité sera accordée aux personnes qui n'ont pas reçu de bourses l'année précédente.

5) Une bourse de voyage ne peut être consentie que si la résidence du requérant ou de la requérante où son employeur ou encore l'université à laquelle il ou elle est rattachê(e) se trouve à plus de 500 $\mathrm{km}$ du lieu où se déroulera le Congrès des Sociétés savantes.

Prière d'adresser toute demande de subvention au Secrétairetrésorier, Association canadienne de science politique, Bureau 205, 1, rue Stewart, Ottawa, Ontario K1N 6H7, avant le $1^{\text {er }}$ février 1997. Les formulaires de demande sont disponibles au bureau de l'Association. 